\title{
Design of Nanowire Optical Cavities as Efficient Photon Absorbers
}

\section{Citation}

Kim, Sun-Kyung, Kyung-Deok Song, Thomas J. Kempa, Robert W. Day, Charles M. Lieber, and Hong-Gyu Park. 2014. "Design of Nanowire Optical Cavities as Efficient Photon Absorbers." ACS Nano (March 13): 140313143802002. doi:10.1021/nn5003776. http://dx.doi.org/10.1021/ nn5003776.

\section{Published Version}

doi: $10.1021 / \mathrm{nn} 5003776$

\section{Permanent link}

http://nrs.harvard.edu/urn-3:HUL.InstRepos:11936450

\section{Terms of Use}

This article was downloaded from Harvard University's DASH repository, and is made available under the terms and conditions applicable to Open Access Policy Articles, as set forth at http:// nrs.harvard.edu/urn-3:HUL.InstRepos:dash.current.terms-of-use\#OAP

\section{Share Your Story}

The Harvard community has made this article openly available.

Please share how this access benefits you. Submit a story.

\section{Accessibility}




\section{Design of Nanowire Optical Cavities as Efficient}

\section{Photon Absorbers}

Sun-Kyung Kim ${ }^{\dagger}$, Kyung-Deok Song ${ }^{*}$, Thomas J. Kempa ${ }^{\S}$, Robert W. Day ${ }^{\S}$, Charles M. Lieber ${ }^{*},, \perp$ and Hong-Gyu Park ${ }^{* * *}$

${ }^{\dagger}$ Department of Applied Physics, Kyung Hee University, Gyeonggi-do 446-701, Republic of Korea, ${ }^{ \pm}$Department of Physics, Korea University, Seoul 136-701, Republic of Korea, ${ }^{\S}$ Department of Chemistry and Chemical Biology and School of Engineering and Applied Sciences, Harvard University, Cambridge, Massachusetts 02138, United States

KEYWORDS core-shell nanowire, solar energy, nanoelectronic device, subwavelength optical cavities, periodic structures, finite-difference time-domain simulations

ABSTRACT Recent investigations of semiconductor nanowires have provided strong evidence for enhanced light absorption, which has been attributed to nanowire structures functioning as optical cavities. Precise synthetic control of nanowire parameters including chemical composition and morphology has also led to dramatic modulation of absorption properties. Here we report finite-difference time-domain (FDTD) simulations for silicon (Si) nanowire cavities to elucidate the key factors that determine enhanced light absorption. The FDTD simulations revealed that a crystalline Si nanowire with an embedded 20-nm-thick amorphous Si shell yields $40 \%$ enhancement of absorption as compared to a homogeneous crystalline Si nanowire, under air-mass 1.5 global solar spectrum for wavelengths between 280 and $1000 \mathrm{~nm}$. Such a large 
enhancement in absorption results from localization of several resonant modes within the amorphous Si shell. A nanowire with a rectangular cross section exhibited enhanced absorption at specific wavelengths with respect to a hexagonal nanowire. The pronounced absorption peaks were assigned to resonant modes with a high symmetry that red-shifted with increasing size of the rectangular nanowire. We extended our studies to investigate the optical properties of singleand multi-layer arrays of these horizontally-oriented nanowire building blocks. The absorption efficiency of a nanowire stack increases with the number of nanowire layers, and was found to be greater than that of a bulk structure or even a single nanowire of equivalent thickness. Lastly, we found that a single-layer nanowire array preserves the structured absorption spectrum of a single nanowire and ascribed this result to a diffraction effect of the periodic nanowire array. The results from these provide insight to the design of nanowire optical cavities with tunable and enhanced light absorption, and thus could help enable the development of ultra-thin solar cells and other nanoscale optoelectronic devices.

The small mode volume, localized resonant modes and long photon lifetimes of subwavelength nanowire (NW) cavities have enabled diverse optoelectronic applications, including ultrasmall light sources, ${ }^{1-5}$ dielectric or plasmonic waveguides, ${ }^{6-9}$ and highly sensitive optical probes. ${ }^{10-11}$ In addition, semiconductor NW photovoltaics (PV) are emerging as a promising platform for the next generation solar cells that require a high efficiency at costs approaching grid parity. ${ }^{12-19}$ In particular, p/i/n core-shell NWs demonstrate electrical and optical properties distinct from conventional planar materials: radial minority carrier separation with short diffusion lengths, and enhanced light absorption resulting from the cavities' subwavelength size. ${ }^{12-14}$ Recently, crystalline Si NW PVs have been successfully demonstrated, 
exhibiting good electrical characteristics as represented by an open-circuit voltage of $\sim 0.5 \mathrm{~V}$ and very low leakage current of $1 \mathrm{fA} .^{13}$ Nevertheless, their relatively low reported current density $\left(J_{\mathrm{SC}}\right)$ of $\sim 7 \mathrm{~mA} / \mathrm{cm}^{2}$ motivates further investigation into strategies for significantly enhancing absorption and corresponding $J_{\mathrm{SC}}$.

Several groups have investigated the interaction of light with individual NWs. ${ }^{12-14,17,20-27}$ For example, absorption or scattering cross sections have been estimated qualitatively, ${ }^{17,20-23}$ while in other works wavelength-dependent photocurrent measurements performed on single NW PV devices were used to assign quantitatively the resonances of NW optical cavities. ${ }^{13-15}$ Concurrent with experiments, numerical and analytical calculations of the resonant modes and key optical figures of merit (e.g. absorption efficiency and scattering cross section) for NW optical cavities have been reported..$^{13,14,20,21,24-27}$ However, systematic studies of design rules for enhancing and tuning light absorption at broad-range wavelengths in NWs and their assembled structures have not been reported. In this study, we address these issues in Si NWs with modulated material compositions and cross-sectional geometries using numerical simulations, and moreover, elucidate the optical properties of single- and multi-layer arrays assembled the NW building blocks.

To accurately describe the optical resonances of NW cavities, we performed threedimensional (3D) finite-difference time-domain (FDTD) calculations. Simulations based on analytical Lorentz-Mie theory and numerical full-field electromagnetic methods have verified experiments and enabled detailed understanding of light-matter interaction in nanoscale optical systems. ${ }^{13,14,20,21}$ Lorentz-Mie theory provides fast and asymptotic solutions of NW optical cavity properties, but is restricted to symmetric structures bounded by a homogeneous medium. ${ }^{20,28}$ In contrast, full-field electromagnetic simulations such as FDTD are not restricted by any 
geometrical constraints and can therefore simulate an experimental NW system with greater accuracy. ${ }^{13,14,21,29}$

In our FDTD simulations, a plane wave with a specific wavelength and polarization (transverse-electric (TE) or transverse-magnetic (TM)) is normally incident to a NW or bulk structure, as shown in Fig. 1 (Methods). The size of NWs used in this study is between 150 to $400 \mathrm{~nm}$. Such subwavelength cavities are characterized by two distinct optical features: highlyconfined resonant modes and an optical antenna effect (Fig. 1a). In contrast, bulk cavities sustain only one-dimensional (1D) Fabry-Perot modes with progressively increasing number of antinodes through the material (Fig. 1b). In this study, we reveal how the distinct optical properties of Si NWs can be harnessed to improve absorption efficiency.

(a)

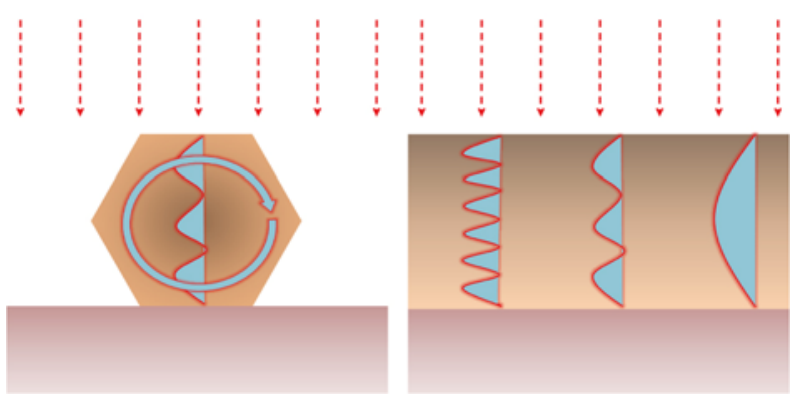

Figure 1. Schematic illustrations showing the interaction between a normally incident plane wave and (a) a NW or (b) a bulk structure.

\section{RESULTS AND DISCUSSION}

Light absorption in core-shell Si NWs. Figure 2a is a representative schematic of the core-shell p/i/n Si NW PV devices studied previously. ${ }^{12-14}$ During illumination by normally incident plane wave, photons couple to specific resonant modes in the NW cavity. These photons are subsequently absorbed by the Si and converted to charge carriers within the embedded p-n 
junction (inset, Fig. 2a). To understand the resonance behavior of NW cavities as a function of the wavelength of incident light, absorption spectra were obtained from FDTD simulations. We examined core-shell NWs homogeneously composed of either crystalline $\mathrm{Si}$ (c-Si) or amorphous $\mathrm{Si}(\mathrm{a}-\mathrm{Si}) .{ }^{30}$ Comparing the absorption spectrum of the c-Si NW to that of the a-Si NW with a c-Si core $(\mathrm{d}=80 \mathrm{~nm})$ reveals several key features that identify how material absorption influences optical resonances in NWs (Fig. 2b). First, the a-Si NW with a c-Si core (solid red, Fig. 2b) has greater absorption efficiency than the c-Si NW (solid black, Fig. 2b) over the entire spectral range except for wavelengths below $450 \mathrm{~nm}$. Second, the spectrum of the a-Si NW with a c-Si core is less structured than that of the c-Si NW. To explain this distinction, we obtained the absorption profiles for both NW structures at a wavelength of $460 \mathrm{~nm}$ (Fig. 2c). The results show that while the c-Si NW sustains a well-defined Fabry-Perot resonance (left, Fig. 2c), the a-Si NW with a c-Si core (right, Fig. 2c) exhibits an attenuation of absorption that decays exponentially from the NW's top surface. The higher optical absorption of a-Si over c-Si leads not only to enhanced absorption efficiency over a broad spectral range, but also to reduced optical feedback within an a-Si, which manifests as reduced peak contrast for corresponding resonant modes. 
(a)

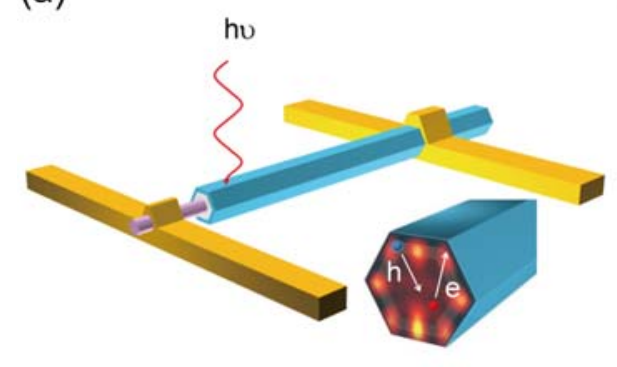

(c)

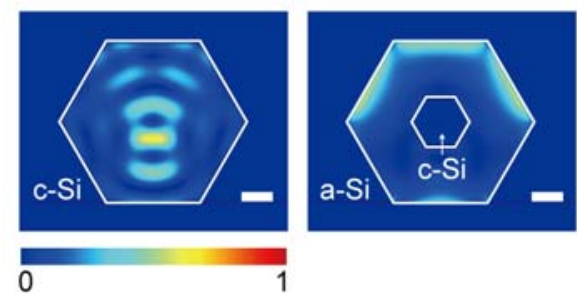

(b)
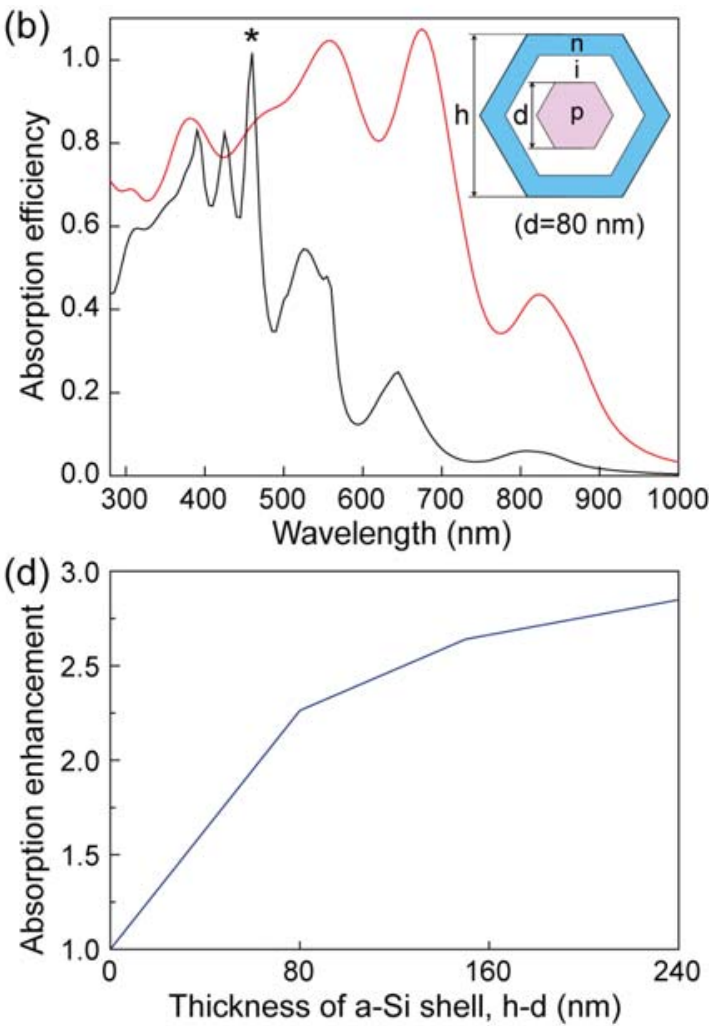

Figure 2. (a) Schematic of a core-shell $\mathrm{p} / \mathrm{i} / \mathrm{n}$ Si NW device converting vertically incident photons into electron-hole pairs. (b) Absorption spectra of a c-Si NW (solid black) and an a-Si NW (solid red) with a NW height (h) of $260 \mathrm{~nm}$. In the a-Si NW, a central p-type core with a height (d) of $80 \mathrm{~nm}$ is treated as c-Si. The inset shows a schematic of the core-shell $\mathrm{p} / \mathrm{i} / \mathrm{n} \mathrm{Si} \mathrm{NW}$. (c) TE absorption mode profiles of the c-Si NW (left) and the a-Si NW with a c-Si core of $\mathrm{d}=80 \mathrm{~nm}$ (right) at a wavelength of $460 \mathrm{~nm}$. The inner boundary in the a-Si NW (right) represents the interface of c-Si core and a-Si shell. The scale bars are $50 \mathrm{~nm}$. (d) Calculated absorption of a-Si NWs with a c-Si core, as a function of the thickness of a-Si shell, h-d, where $\mathrm{h}$ is fixed to be 260 $\mathrm{nm}$. The value is normalized by the absorption of a c-Si NW with the same height of $260 \mathrm{~nm}$.

Next, we calculated the total absorption of a NW composed of a c-Si core and an a-Si shell by integrating its absorption efficiency over air-mass 1.5 global (AM 1.5 G) solar spectrum 
(280 - $1000 \mathrm{~nm}$ ), while varying the thickness of the a-Si shell, h-d (Fig. 2d) (Methods). The absorption of this NW increases monotonically as the volume fraction of a-Si increases. The relative enhancement at an a-Si shell thickness of $160 \mathrm{~nm}$ is $\sim 2.6$, which explains well the difference of current density $\left(J_{\mathrm{SC}}\right)$ of previously reported c-Si and nanocrystalline $\mathrm{Si}$ (nc-Si) devices. ${ }^{12,13}$ For this comparison, we postulate that the absorption dispersion of nc-Si is similar to that of a-Si. It is important to note that although an nc-Si NW yields higher $J_{\mathrm{SC}}$ than a c-Si NW, the nc-Si material may preclude further enhancement of $J_{\mathrm{SC}}$ through tuning of resonant modes. Furthermore, the grain-boundaries intrinsic to nc-Si is detrimental to the electrical performance and power conversion efficiency of reported NW PV devices. ${ }^{12}$ Indeed, the poor $V_{\mathrm{OC}}$ of nc-Si devices compensates for their overall higher absorption.

Multi-shell Si NW with an a-Si inner shell. To minimize electrical degradation due to nc-Si while taking advantage of its high absorption, we designed a multi-shell NW composed of c-Si with a thin embedded nc-Si layer. ${ }^{26,31}$ For these calculations, we treat nc-Si in the thin embedded layer as a-Si. In the multi-shell NW, we introduced a 20-nm-thick a-Si layer at a height position of h'=105 nm within a c-Si NW with a total height of h=220 nm (inset, Fig. 3a). The absorption spectrum of the multi-shell NW (solid red, Fig. 3a) reveals several important features when compared to a homogeneous c-Si NW of equivalent height (solid black, Fig. 3a). First, both spectra show similar peak amplitudes at ultraviolet to blue wavelengths, but at wavelengths longer than $450 \mathrm{~nm}$ the multi-shell NW yields significantly enhanced absorption efficiency. Second, the multi-shell NW still exhibits a structured spectrum with many distinct absorption peaks. The wavelength of each resonant peak is nearly the same as that of the c-Si NW. To better understand the absorption features of a multi-shell NW, we calculated absorption (Fig. 3b) and electric-field intensity profiles (Fig. 3c). At $380 \mathrm{~nm}$ (left, Fig. 3b), no absorption 
takes place in the a-Si layer, because most of the incident light is absorbed in the top c-Si layer. In contrast, at $530 \mathrm{~nm}$ (right, Fig. 3b), significant absorption is observed in the a-Si layer giving rise to the absorption enhancement witnessed for longer wavelengths (Fig. 3a). Notably, the electric field intensity profile for the given mode is preserved (left, Fig. 3c) as compared to a c-Si NW (right, Fig. 3c), accounting for the preservation of resonant wavelengths (Fig. 3a).

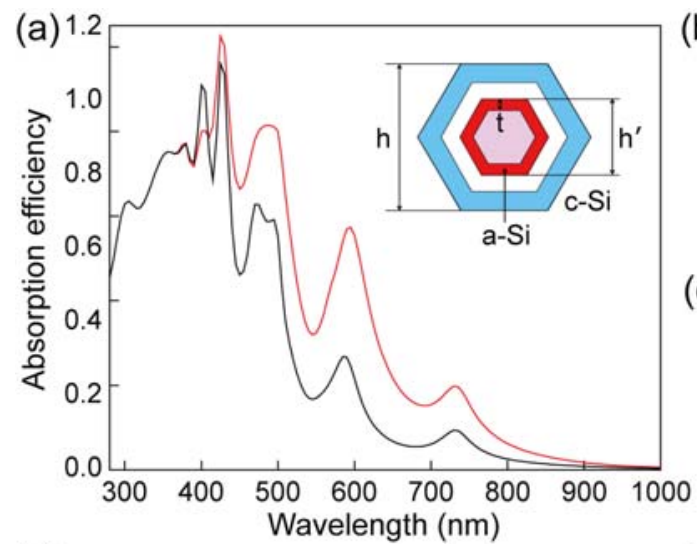

(b)
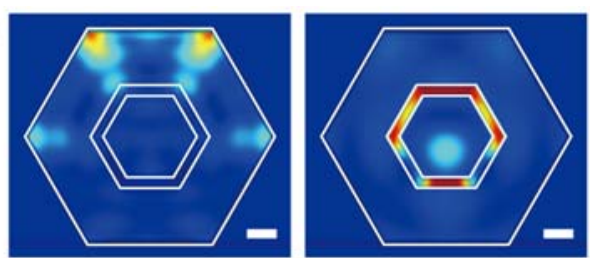

(c)
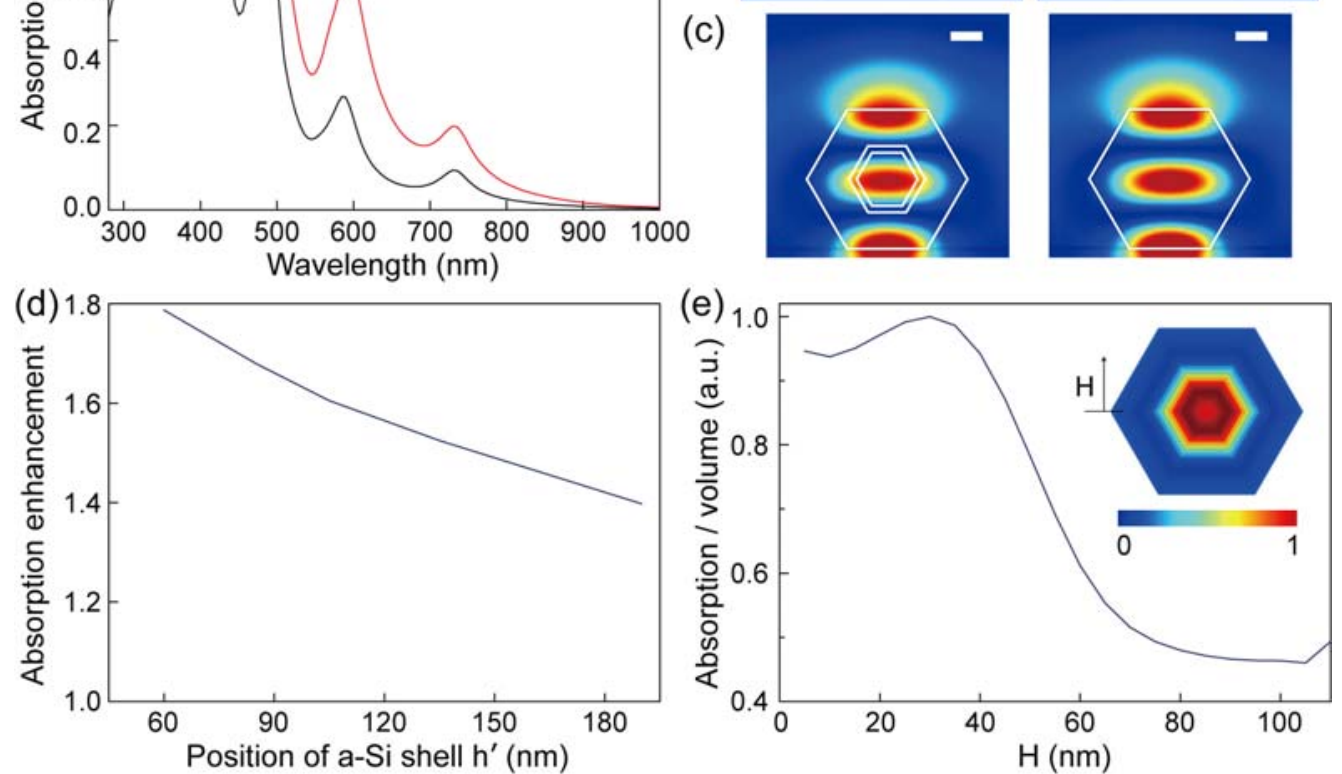

Figure 3. (a) Absorption spectrum of the multi-shell NW ( $h=220 \mathrm{~nm}$ ) with an a-Si inner shell ( $\mathrm{t}=20 \mathrm{~nm}$ ) (solid red). The height position of a-Si shell (h') is $105 \mathrm{~nm}$. For a reference, the absorption spectrum of a c-NW with $\mathrm{h}=220 \mathrm{~nm}$ is plotted together (solid black). (b) TM absorption mode profiles of the multi-shell NW of (a) at $\lambda=380 \mathrm{~nm}$ (left) and $\lambda=470 \mathrm{~nm}$ (right). The scale bars are $30 \mathrm{~nm}$. (c) TM electric field intensity profiles of the multi-shell NW (left) and the c-Si NW (right) at $\lambda=730 \mathrm{~nm}$. The scale bars are $50 \mathrm{~nm}$. (d) Calculated absorption of the multi-shell NW with $\mathrm{h}=220 \mathrm{~nm}$ as a function of the position of an a-Si inner shell, h'. The value 
is normalized by the absorption of a c-Si NW with the same height of $220 \mathrm{~nm}$. (e) Calculated absorption per unit volume generated from every 10 -nm-thick inner shell while increasing the height of the inner shell, $\mathrm{H}$. The inset shows a 2D representation of the spatial absorption distribution. For this calculation, a c-Si NW with $\mathrm{h}=220 \mathrm{~nm}$ is used.

Next, we calculated the absorption of a multi-shell NW while moving the position of the a-Si layer (Fig. 3d) from core to shell. For these calculations, the thickness of the a-Si layer was adjusted to preserve area. The result showed that the absorption of a multi-shell NW is enhanced steadily as the embedded a-Si layer is shifted to the core. The maximum enhancement in absorption can be as large as 1.8 compared to a homogeneous c-Si NW. To understand the absorption behavior depending on the position of a-Si layer, we calculated the internal absorption distribution of the NW (Fig. 3e) (Methods). Distinct from a bulk structure, a NW exhibits highly localized absorption in the core $(\mathrm{H} \sim 50 \mathrm{~nm})$ and this effect, when paired with a thin highly absorptive layer such as a-Si, leads to a large enhancement of absorption and also the observed trend in Fig. 3d. Notably, the thin a-Si layer contributes to significantly enhanced absorption at long wavelengths $(600-800 \mathrm{~nm})$ where c-Si has small absorption coefficient (Fig. 3a and Supplementary Fig. S1). Taken together, manipulation of the internal absorption modes in a NW cavity provides a new route to enhance light absorption and to enable more efficient photon absorbers. For an actual c-Si/a-Si NW PV device, a transparent conductive oxide layer composed of ITO or $\mathrm{ZnO}: \mathrm{Al}$ surrounding the outer shell of the NW may be necessary to improve carrier collection efficiency due to enhanced recombination in the a-Si shell. ${ }^{32}$ We note that addition of such transparent layers could also function as antireflective coatings for increased light absorption. ${ }^{32}$ 
NWs with hexagonal and rectangular cross sections. We also investigated the absorption properties of Si NWs with different cross-sectional geometries. ${ }^{14,20}$ Chemical vapor deposition (CVD) can yield crystalline Si NWs with various cross-sectional geometries by controlling several growth parameters. ${ }^{14,33}$ Recently, it was reported that n-type Si shell growth at elevated temperatures leads to accelerated growth rates over specific facets of crystalline $\mathrm{Si}$ NWs to transform hexagonal cross section into rectangular one. ${ }^{14}$ In our study, we considered hexagonal and rectangular NWs with the same aspect ratio (height-to-width ratio) of 0.87 (Fig. 4a). 

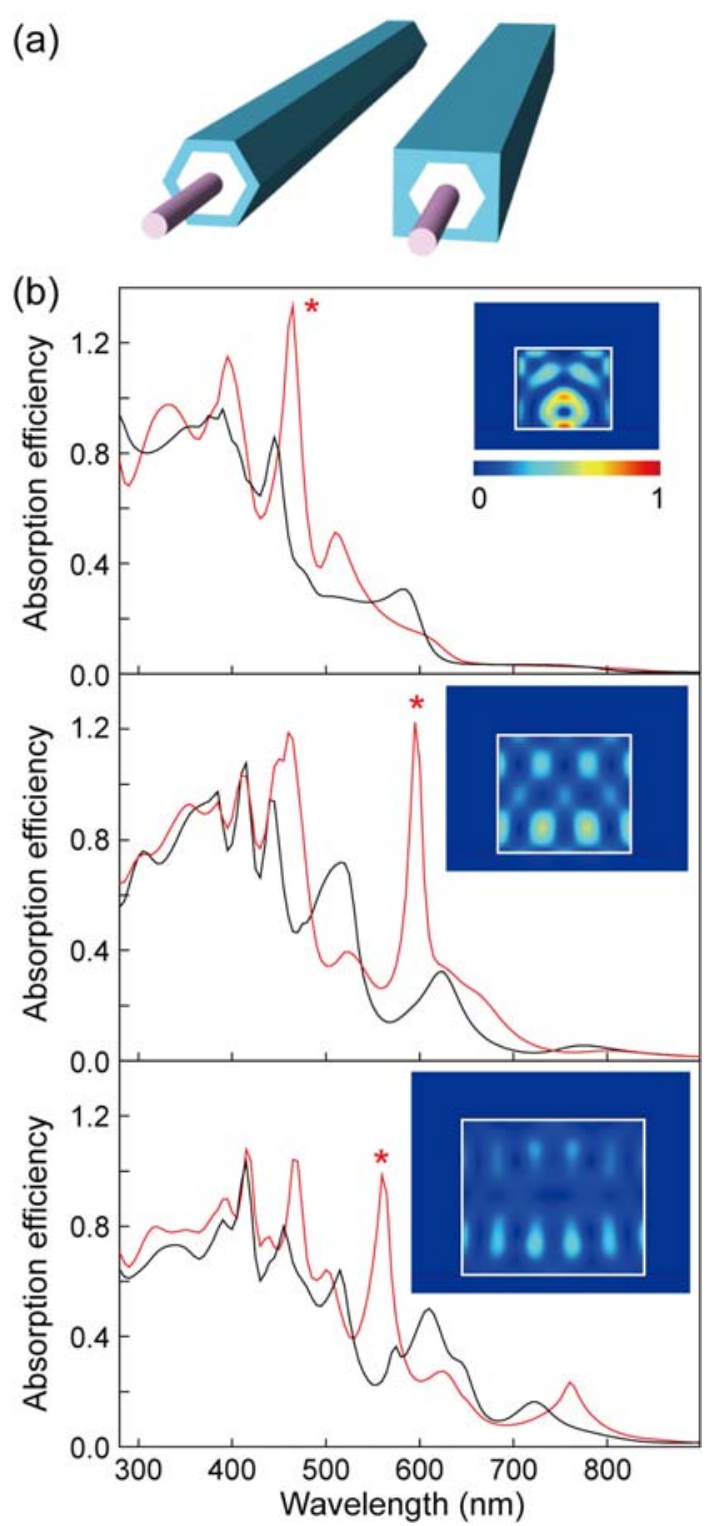

Figure 4. (a) Schematics of p/i/n Si NWs with hexagonal (left) and rectangular (right) crosssectional geometry. (b) TE absorption spectra of hexagonal (solid black) and rectangular (solid red) NWs with heights of 160 (top), 240 (middle) and $320 \mathrm{~nm}$ (bottom). The inset shows absorption profile of the peak marked by * in each spectrum.

We calculated the TE absorption spectra of hexagonal and rectangular NWs with heights of $160 \mathrm{~nm}$ (top, Fig. 4b), $240 \mathrm{~nm}$ (middle, Fig. 4b) and $320 \mathrm{~nm}$ (bottom, Fig. 4b). Comparison of 
both structures with the same heights reveals that rectangular NWs have enhanced absorption peaks at specific wavelengths: for example, $465 \mathrm{~nm}$ from the smallest NW, $595 \mathrm{~nm}$ from the intermediate NW and $560 \mathrm{~nm}$ from the largest NW. The pronounced absorption peaks are assigned to highly symmetric resonant modes (inset, Fig. 4b) that are not excited in a hexagonal NW due to symmetry mismatch. The highly symmetric modes in rectangular NWs exhibit a larger quality factor ${ }^{14}$ and mode volume that ultimately leads to higher-amplitude absorption peaks at specific wavelengths. Despite the relatively thin Si absorber in a rectangular NW, a peak at $\sim 600 \mathrm{~nm}$ exhibits amplitude over unity. We conclude that tuning of high absorption peaks from a rectangular NW may enable an efficient wavelength-specific photodetector.

Vertical NW stacks. We have proposed several strategies and design rules to improve light absorption by single NWs over a broad range of wavelengths. However, to produce significant electrical power outputs comparable to those of large area solar panels, it is necessary to scale up a vast number of NWs in vertical and/or horizontal planes. Therefore, we have also investigated the optical properties of periodic arrays of NW elements and calculated their $J_{\mathrm{SC}}$ 's to examine the potential of NWs in future photovoltaics.

First, we consider layered vertical stacks of NWs and compare their efficiency to bulk structures or single NWs with equivalent $\mathrm{Si}$ thicknesses (Fig. 5a). ${ }^{13}$ Figure $5 \mathrm{~b}$ shows the calculated $J_{\mathrm{SC}}$ of bulk Si (solid black), a single NW (solid red) and a vertical NW stack (solid blue) under AM $1.5 \mathrm{G}$ 1-sun $\left(100 \mathrm{~mW} / \mathrm{cm}^{2}\right)$ illumination as a function of increasing Si thickness, assuming unity internal quantum efficiency. Comparison of the $J_{\mathrm{SC}}$ produced by each structure highlights several key features. First, a vertical NW stack yields a higher $J_{\mathrm{SC}}$ than either bulk $\mathrm{Si}$ or a single NW of the same thickness. In particular, an approximately $1-\mu \mathrm{m}$-thick vertical NW stack composed of four identical NWs produces a $J_{\mathrm{SC}}$ of $\sim 18.3 \mathrm{~mA} / \mathrm{cm}^{2}$, which is $\sim 120 \%(\sim 15 \%)$ 
larger than bulk Si (a single NW) of the same thickness. Second, the $J_{\mathrm{SC}}$ of a vertical NW stack increases linearly up to a $\mathrm{Si}$ thickness of $1 \mu \mathrm{m}$ while bulk structures and a single NW show relatively sub-linear increases in $J_{\mathrm{SC}}$ over the same $\mathrm{Si}$ thickness. In other words, the enhancement in $J_{\mathrm{SC}}$ from a vertical NW stack compared to the other structures becomes more noticeable as $\mathrm{Si}$ thickness increases.
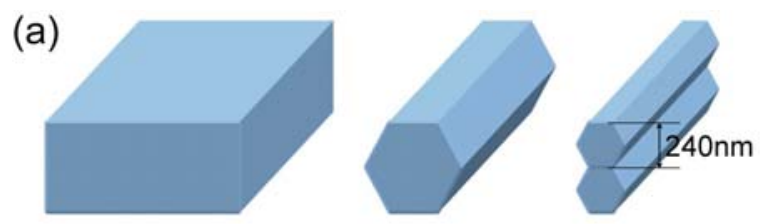

(b)
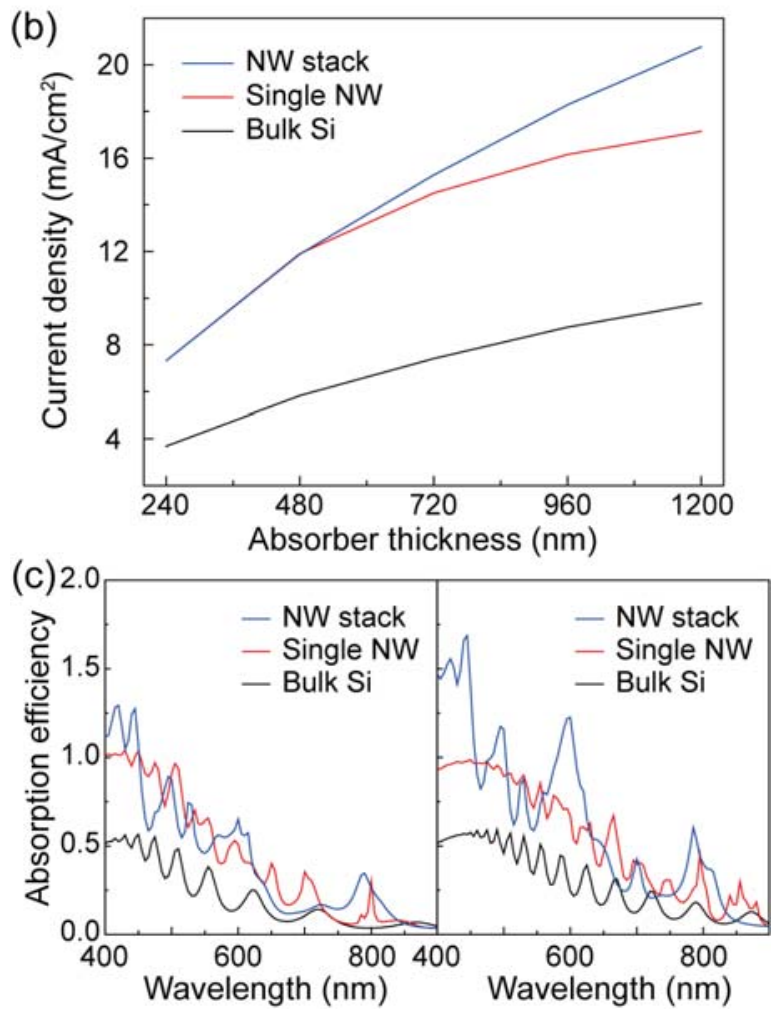

Figure 5. (a) Schematics of a bulk structure (left), a single NW (middle), and a vertical NW stack consisting of two identical NWs (right). (b) Calculated current densities of a bulk structure (solid black), a single NW (solid red), and a vertical NW stack consisting of NWs with a height of $240 \mathrm{~nm}$ (solid blue) under 1-sun illumination as a function of the Si thickness, assuming unity internal quantum efficiency. For the vertical NW stack, the current densities of one to five NW 
stacks are plotted. All simulated NW structures consist of homogeneous crystalline Si. (c) Absorption spectra of a bulk structure (solid black), a single NW (solid red), and a vertical NW stack consisting of NWs with a height of $240 \mathrm{~nm}$ (solid blue). The total Si thickness is $480 \mathrm{~nm}$ (left) and $960 \mathrm{~nm}$ (right).

To better understand the physical origins of the trend of $J_{\mathrm{SC}}$ as a function of Si thickness, we considered the absorption spectra of bulk $\mathrm{Si}$, a single NW, and a vertical NW stack at $\mathrm{Si}$ thicknesses of $480 \mathrm{~nm}$ (left, Fig. 5c) and $960 \mathrm{~nm}$ (right, Fig. 5c). For a vertical NW stack, peak amplitude is enhanced with a nearly constant factor over the range of wavelengths considered and this accounts for the large $J_{\mathrm{SC}}$ and also the linear increase of $J_{\mathrm{SC}}$ with increasing Si thickness. This broadband enhancement stems from reduced screening of NWs by their nearest neighbors due to the optical antenna effect experienced by every NW in the stack. ${ }^{13}$ Taken together, a vertical NW stack represents a new building block for assembly of efficient NW solar cells. Such stacks surpass the light absorption of a single bulk structure or a single NW, and further enhancements may be possible by employing appropriate combinatorial sets of NWs composed of different materials, sizes, or cross-sectional geometries.

Close-packed NW array. Next, we investigated the properties of a close-packed horizontal NW array (inset, Fig. 6a). We calculated the $J_{\mathrm{SC}}$ of a single NW (solid black, Fig. 6a) and NW array (red point, Fig. 6a) under 1-sun illumination as a function of Si thickness, assuming unity internal quantum efficiency. The results show that the $J_{\mathrm{SC}}$ of a single NW with a Si thickness of $150-400 \mathrm{~nm}$ is nearly preserved even when scaled up to a NW array. We note that the NW optical antenna effect is suppressed in an array. To illustrate what causes the NW array and single NW to have very similar $J_{\mathrm{SC}}$, we calculated the absorption spectra for both 
structures with a Si thickness of $240 \mathrm{~nm}$ (Fig. 6b). The result shows that every absorption peak within the NW array spectrum has the similar wavelength and amplitude to that within the single NW spectrum. On the other hand, polarization-resolved absorption spectra of a NW array are distinct from those of a single NW (Supplementary Fig. S2). Specifically, light absorption within the NW array is enhanced for TE-polarized incident light (electric-field oscillation direction parallel to the surface modulation of the NW array). We conclude that the optical antenna effect experienced by a single NW is attenuated but compensated for by a diffraction effect caused by the periodic surface modulation of the NW array. ${ }^{20,34}$ 

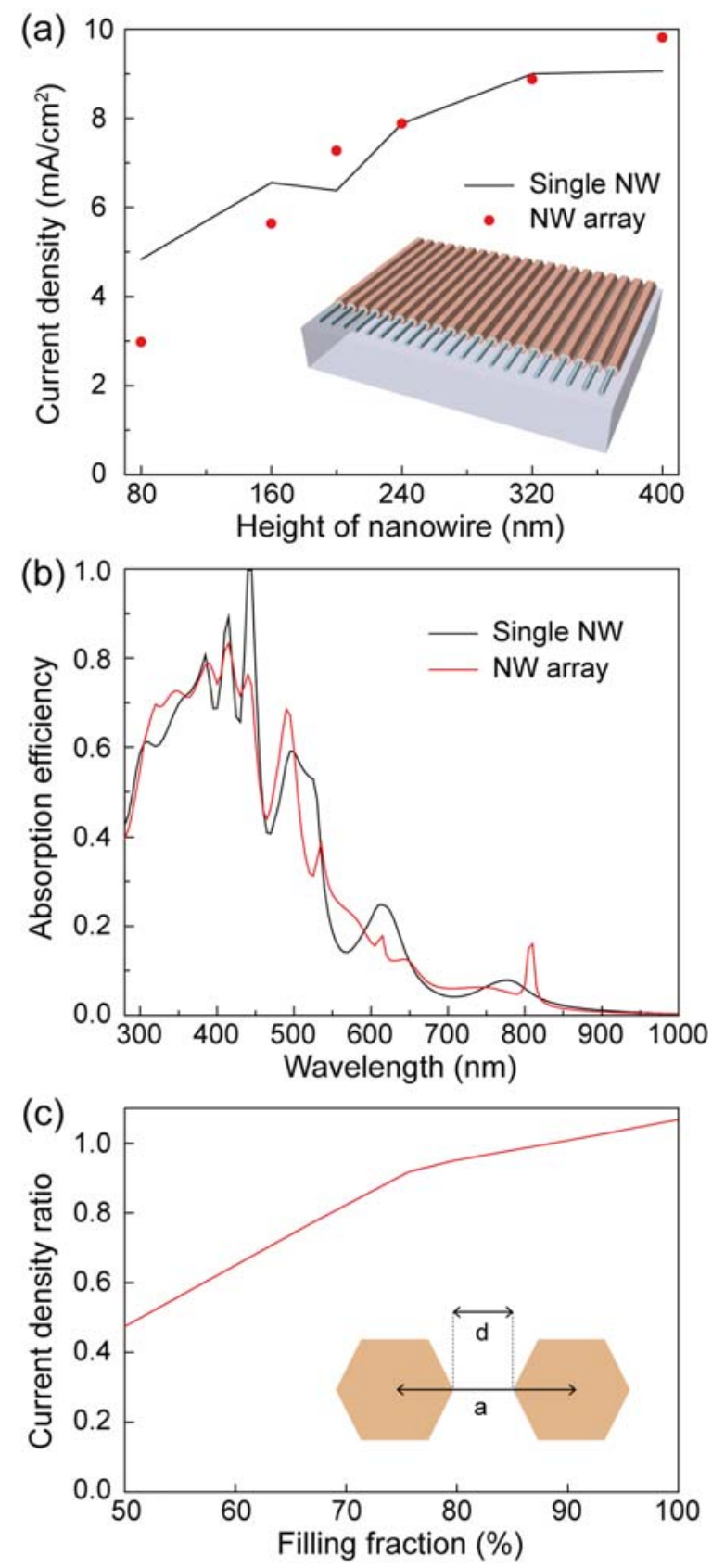

Figure 6. (a) Calculated current densities of a single NW (solid black) and a close-packed NW array (red point) under 1-sun illumination as a function of the height of a NW, assuming unity internal quantum efficiency. All simulated NW structures consist of homogeneous crystalline Si. The inset shows a schematic of a close-packed NW array. (b) Absorption spectra of a single NW (solid black) and a close-packed NW array (solid red) with a Si thickness of $240 \mathrm{~nm}$. (c) 
Calculated current density of a NW array as a function of the filling fraction, 1-(d/a) (inset). The current density of the NW array is normalized by the current density of a single NW.

To predict the performance of more realistic NW array structures, we calculated the absorption efficiency of a NW array depending on its filling fraction. The ratio of the $J_{\mathrm{SC}}$ of a NW array to that of a single NW was plotted while varying the filling fraction of the NW array (Fig. 6c). The filling fraction is defined as 1-(d/a), where a is the pitch size and $\mathrm{d}$ is the distance between each NW (inset, Fig. 6c). For purposes of calculating $J_{\mathrm{SC}}$, we define the area of a NW array as its total projected area including Si projected area and air space. Notably, the $J_{\mathrm{SC}}$ of a NW array is reduced only by $\sim 10 \%$ even at a filling fraction of $75 \%$. Interestingly, if we consider only the Si projected area, the $J_{\mathrm{SC}}$ of a NW array (at $75 \%$ filling fraction) is $20 \%$ higher than that of a single NW. We infer that the diffraction effect of the NW array is enhanced at specific pitch sizes. ${ }^{20,34}$ We conclude that the absorption efficiency of a single NW is nearly preserved in NW arrays and even in those with sizeable void spaces. These observations shed light on implementation of large area arrays of NW PVs for next generation solar cells. ${ }^{35}$

\section{CONCLUSIONS}

In summary, we investigated and provided design rules for enhancement of optical absorption by Si NW cavities through the changes in morphology and core/shell composition. Si NWs with modulated material composition (c-Si and a-Si polymorphic structures) and crosssectional geometry show substantial enhancements in absorption efficiency over a broad range of wavelengths. Our studies reveal that the increased light absorption can be attributed to localized resonant modes and optical antenna effects of NW cavities. In addition, these investigations have 
shown that vertically-stacked NWs have larger absorption efficiency than both a single NW and a bulk structure of equivalent thickness. The absorption efficiency of a single NW is nearly preserved when it is extended to a horizontal NW array, because a diffraction effect of the array compensates for attenuation of the optical antenna effect. These findings will be useful to design NW structures and arrays with improved absorption properties, and thus will help in experimental evaluations of these materials as possible next generation solar cells and efficient nanophotonic components.

\section{METHODS}

Numerical FDTD calculations. We used our in-house FDTD code for all NW optical simulations. ${ }^{36}$ First, while a plane wave with TE or TM polarization is normally incident to a $\mathrm{NW}$, the absorption cross section of the NW is calculated by integrating $J \cdot \mathrm{E}$ at each grid point over one optical cycle, where $J$ and $\mathrm{E}$ are the polarization current density and electric field, respectively. Then, the absorption cross section was divided by the projected area of the NW to yield the absorption efficiency. For calculating $J_{\mathrm{SC}}$, internal quantum efficiency (IQE) is assumed to be unity. To describe NWs with a hexagonal (rectangular) cross section, a spatial resolution of $5 / \sqrt{3}(5), 5$, and $5 \mathrm{~nm}$ is used along $\mathrm{x}-, \mathrm{y}$ - and z-axes, respectively, where $\mathrm{y}$-axis lies along the NW axis and z-axis lies along the propagation direction of the incident plane wave. For calculations on single NWs, periodic boundary condition is applied along y-axis and additionally, total-field scattered-field method is used. For calculations on NW array structures, periodic boundary condition is applied along $\mathrm{x}$ - and y-axes. The total $J_{\mathrm{SC}}$ is calculated by integrating $J_{\mathrm{SC}}(\lambda)$ over the wavelength range of $280-1000 \mathrm{~nm}$, where $J_{\mathrm{SC}}(\lambda)=\operatorname{absorption}$ efficiency $(\lambda) \times$ 
IQE $\times($ spectral irradiance of AM $1.5 \mathrm{G}$ spectrum at 1 -sun solar intensity $) \times \lambda / 1.24$. For calculating spatial absorption distribution of the hexagonal NW $(\mathrm{h}=220 \mathrm{~nm})$, absorption is calculated within a hexagonal shell with a thickness of $10 \mathrm{~nm}$ while the height of the hexagonal shell increase from 10 to $220 \mathrm{~nm}$.

\section{ASSOCIATED CONTENT}

\section{Supporting Information}

Additional figures. This material is available free of charge via the Internet at http://pubs.acs.org.

\section{AUTHOR INFORMATION}

\section{Corresponding Author}

*E-mail: $\underline{\text { cml@ @mliris.harvard.edu, hgpark@korea.ac.kr }}$

\section{Author Contributions}

The manuscript was written through contributions of all authors. All authors have given approval to the final version of the manuscript.

\section{ACKNOWLEDGMENT}

H.-G.P. acknowledges support of this work by the National Research Foundation of Korea (NRF) grant funded by the Korea government (MSIP) (No. 2009-0081565). C.M.L. acknowledges support of this work by the National Science Foundation under NSF award no. ECS-0335765. S.-K.K. acknowledges support of this work by Basic Science Research Program through the National Research Foundation of Korea (NRF) funded by the Ministry of Science, ICT \& Future Planning (NRF-2013R1A1A1059423). T.J.K. acknowledges the support of a 
National Science Foundation Graduate Research Fellowship. R.W.D. acknowledges a NSF Graduate Research Fellowship.

\section{ABBREVIATIONS}

NW, nanowire; FDTD, finite-difference time-domain; PV, photovoltaic; TE, transverse-electric;

$\mathrm{TM}$, transverse-magnetic; a-Si, amorphous $\mathrm{Si}$; c-Si, crystalline $\mathrm{Si}$; EQE, external quantum efficiency; IQE, internal quantum efficiency

\section{REFERENCES AND NOTES}

(1) Qian, F.; Li, Y.; Gradecak, S.; Park, H.-G.; Dong, Y.; Ding, Y.; Wang, Z. L.; Lieber, C. M. Multi-Quantum-Well Nanowire Heterostructures for Wavelength-Controlled Lasers. Nat. Mater. 2008, 7, 701-706.

(2) Chu, S.; Wang, G.; Zhou, W.; Lin, Y.; Chernyak, L.; Zhao, J.; Kong, J.; Li, L.; Ren, J.; Liu, J. Electrically Pumped Waveguide Lasing from ZnO Nanowires. Nat. Nanotech. 2011, 6, 506510.

(3) Oulton, R. F.; Sorger, V. J.; Zentgraf, T.; Ma, R.-M.; Gladden, C.; Dai, L.; Bartal. G.; Zhang, X. Plasmon Lasers at Deep Subwavelength Scale. Nature 2009, 461, 629-632.

(4) Barrelet, C. J.; Ee, H.-S.; Kwon, S.-H.; Park, H.-G. Nonlinear Mixing in Nanowire Subwavelength Waveguides. Nano Lett. 2011, 11, 3022-3025.

(5) Cao, L. Y.; Park, J. S.; Fan, P. Y.; Clemens, B.; Brongersma, M. L. Epitaxial Growth of InGaN Nanowire Arrays for Light Emitting Diodes. ACS Nano 2011, 5, 3970-3976.

(6) Pyayt, A. L.; Wiley, B.; Xia, Y.; Chen, A.; Dalton. L. Integration of Photonic and Silver Nanowire Plasmonic Waveguides. Nat. Nanotech. 2008, 3, 660-665. 
(7) Park, H.-G.; Barrelet, C. J.; Wu, Y.; Tian, B.; Qian, F.; Lieber, C. M. A Wavelength-

Selective Photonic-Crystal Waveguide Coupled to a Nanowire Light Source. Nat. Photon. 2008, 2, 622-626.

(8) Barrelet, C. J.; Bao, J.; Loncar, M.; Park, H.-G.; Capasso, F.; Lieber, C. M. Hybrid SingleNanowire Photonic Crystal and Microresonator Structures. Nano Lett. 2006, 6, 11-15.

(9) Zhao, Y. S.; Zhan, P.; Kim. J.; Sun, C.; Huang. J. Patterned Growth of Vertically Aligned Organic Nanowire Waveguide Arrays. ACS Nano. 2010, 4, 1630-1636.

(10) Nakayama, Y.; Pauzauskie, P. J.; Radenovic, A.; Onorato, R. M.; Saykally, R. J.; Liphardt, J.; Yang, P. Tunable Nanowire Nonlinear Optical Probe. Nature 2007, 447, 1098-1101.

(11) Gu, F.; Zhang, L.; Yin, X.; Tong, L. Polymer Single-Nanowire Optical Sensors. Nano Lett. 2008, 8, 2757-2761.

(12) Tian, B. Z.; Zheng, X.; Kempa, T. J.; Fang, Y.; Yu, N.; Yu, G.; Huang, J.; Lieber, C. M. Coaxial Silicon Nanowires as Solar Cells and Nanoelectronic Power Sources. Nature 2007, 449, $885-889$.

(13) Kempa, T. J.; Cahoon, J. F.; Kim, S.-K.; Day, R. W.; Bell, D. C.; Park. H.-G.; Lieber, C. M. Coaxial Multishell Nanowires with High-Quality Electronic Interfaces and Tunable Optical Cavities for Ultrathin Photovoltaics. Proc. Natl. Acad. Sci. USA. 2012, 109, 1409-1412.

(14) Kim, S.-K.; Day, R. W.; Cahoon, J. F.; Kempa, T. J.; Song, K.-D.; Park, H.-G.; Lieber, C. M. Tuning Light Absorption in Core/Shell Silicon Nanowire Photovoltaic Devices through Morphological Design. Nano Lett. 2012, 12, 4971-4976.

(15) Tang, J.; Huo, Z.; Brittman, S.; Yang, P. Solution-Processed Core-Shell Nanowires for Efficient Photovoltaic Cells. Nat. Nanotech. 2011, 6, 568-572. 
(16) Wallentin, J.; Anttu, N.; Asoli, D.; Huffman, M.; Åberg, I.; Magnusson, M. H.; Siefer, G.; Fuss-Kailuweit, P.; Dimroth, F.; Witzigmann, B.; et al. InP Nanowire Array Solar Cells Achieving 13.8\% Efficiency by Exceeding the Ray Optics Limit. Science 2013, 339, 1057-1060. (17) Kelzenberg, M. D.; Turner-Evans, D. B.; Putnam, M. C.; Boettcher, S. W.; Briggs, R. M.; Baek, J. Y.; Lewis, N. S.; Atwater, H. A. High-Performance Si Microwire Photovoltaics. Energy Environ. Sci. 2011, 4, 866-871.

(18) Mariani, G.; Wong, P-S.; Katzenmeyer, A. M.; Leonard, F.; Shapiro, J.; Huffaker, D. L.; Patterned Radial GaAs Nanopillar Solar Cells. Nano Lett. 2011, 11, 2490-2494.

(19) Fan, Z.; Razavi, H.; Do, J.-W.; Moriwaki, A.; Ergen, O.; Chueh, Y-L.; Leu, P. W.; Ho, J. C.; Takahashi, T.; Reichertz, L. A.; et al. Three-Dimensional Nanopillar-Array Photovoltaics on Low-Cost and Flexible Substrates. Nat. Mater. 2009, 8, 648-653.

(20) Cao, L.; Fan, P.; Vasudev, A. P.; White, J. S.; Yu, Z.; Cai, W.; Schuller, J. A.; Fan, S.; Brongersma, M. L. Semiconductor Nanowire Optical Antenna Solar Absorbers. Nano Lett. 2010, $10,439-445$.

(21) Barnard, E. S.; Pala, R. A.; Brongersma, M. L. Photocurrent Mapping of Near-Field Optical Antenna Resonances. Nat. Nanotech. 2011, 6, 588-593.

(22) Giblin, J.; Syed, M.; Banning, M. T.; Kuno, M.; Hartland, G. Experimental Determination of Single CdSe Nanowire Absorption Cross Sections through Photothermal Imaging. ACS Nano 2010, 4, 358-364.

(23) Bronstrup, G.; Leiterer, C.; Jahr, N.; Gutsche, C.; Lysov, A.; Regolin, T.; Prost, W.; Tegude, F. J.; Fritzsche, W.; Christiansen, S. A Precise Optical Determination of Nanoscale Diameters of Semiconductor Nanowires. Nanotechnology 2011, 22, 382501/1-9. 
(24) Bronstrup, G.; Jahr, N.; Leiterer, C.; Csaki, A.; Fritzsche, W.; Christiansen, S. Optical Properties of Individual Silicon Nanowires for Photonic Devices. ACS Nano 2010, 4, 71137122.

(25) Liu, W. F.; Oh, J. I.; Shen, W. Z. Light Trapping in Single Coaxial Nanowires for Photovoltaic Applications. IEEE Electron Device Lett. 2011, 32, 45-47.

(26) Liu, W. F.; Oh, J. I.; Shen, W. Z. Light Absorption Mechanism in Single c-Si (core)/a-Si (shell) Coaxial Nanowires. Nanotechnology 2011, 22, 125705-125708.

(27) Yu, Y.; Ferry, V. E.; Alivisatos, A. P.; Cao, L. Dielectric Core-Shell Optical Antennas for Strong Solar Absorption Enhancement. Nano Lett. 2012, 12, 3674-3681.

(28) Bohren, C. F.; Huffman, D. R. Absorption and Scattering of Light by Small Particles. New York: Wiley 1998.

(29) Taflove, A.; Hagness, S. C. Computational Electrodynamics: The Finite-Difference TimeDomain Method. Artech House: Norwood, MA 2005.

(30) Lide, D. R. CRC handbook of chemistry and physics: a ready-reference book of chemical and physical data. CRC Press, Boca Raton 2008.

(31) Adachi, M. M.; Anantram, M. P.; Karim, K. S. Optical Properties of Crystalline Amorphous Core-Shell Silicon Nanowires. Nano Lett. 2010, 10, 4093-4098.

(32) Deceglie, M. G.; Ferry, V. E.; Alivisatos, A. P.; Atwater, H. A. Design of Nanostructured Solar Cells Using Coupled Optical and Electrical Modeling. Nano Lett. 2012, 12, 2894-2900.

(33) Cho, B.; Bareno, J.; Foo, Y. L.; Hong, S.; Spila, T.; Petrov, I.; Greene, J. E. Phosphorus Incorporation during Si(001): P Gas-source Molecular Beam Epitaxy: Effects on Growth Kinetics and Surface Morphology. J. Appl. Phys. 2008, 103, 123530/1-10 . 
(34) Yu, Z.; Raman, A.; Fan, S. Fundamental Limit of Nanophotonic Light Trapping in Solar Cells. Proc. Natl. Acad. Sci. USA 2010, 107, 17491-17496.

(35) Javey, A.; Nam, S.; Friedman, R. S.; Yan, H.; Lieber, C. M. Layer-by-Layer Assembly of Nanowires for Three-Dimensional, Multifunctional Electronics. Nano Lett. 2007, 7, 773-777.

(36) Ee, H.-S.; Song, K.-D.; Kim, S.-K.; Park, H.-G. Finite-Difference Time-Domain

Algorithm for Quantifying Light Absorption in Silicon Nanowires. Isr. J. Chem. 2012, 52, 10271036. 
ToC graphic

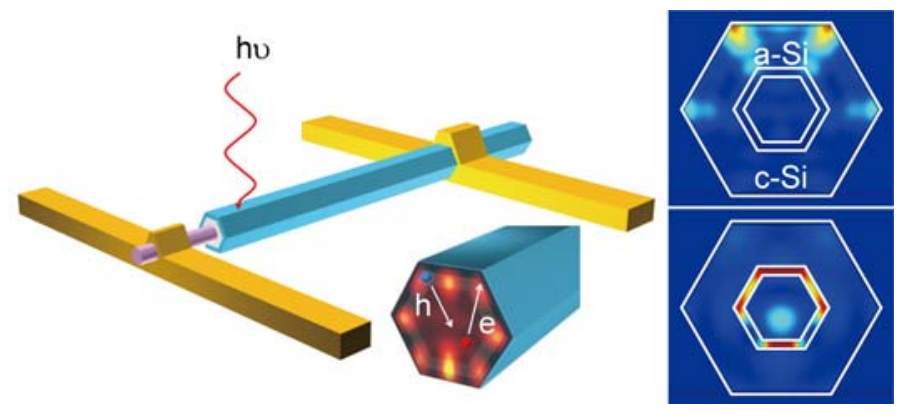


Supporting Information for:

\section{Design of Nanowire Optical Cavities as Efficient Photon Absorbers}

Sun-Kyung Kim, Kyung-Deok Song, Thomas J. Kempa, Robert W. Day, Charles M. Lieber, and Hong-Gyu Park

This file includes:

Supplementary Figures S1 and S2 


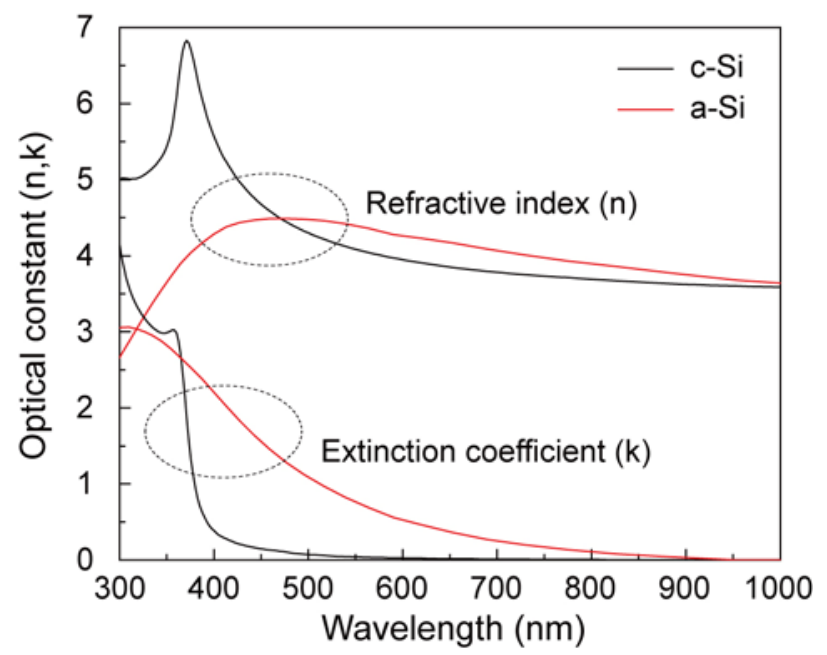

Figure S1. Dispersion relations of c-Si and a-Si, used in Si NW simulations.
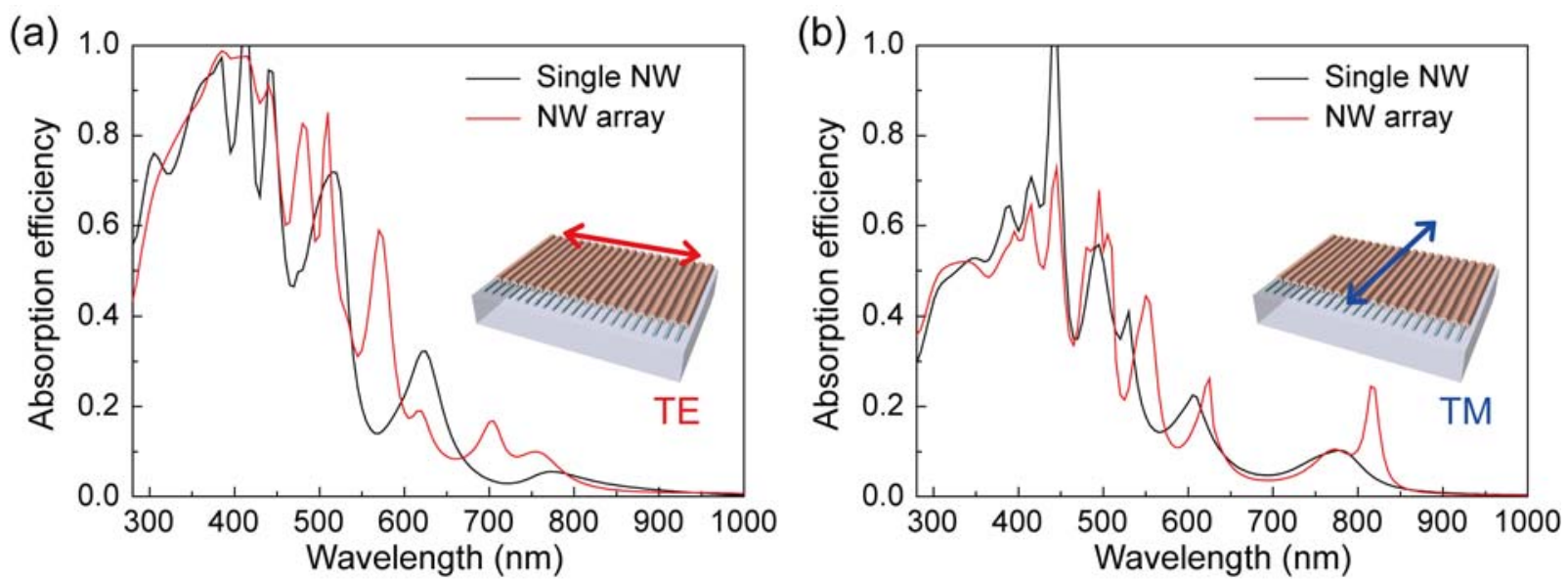

Figure S2. Calculated TE-polarized (a) and TM-polarized (b) absorption spectra of a single NW and a NW array with an equivalent NW height of $240 \mathrm{~nm}$. 